\title{
A RANDOMIZED CLINICAL TRIAL COMPARING ARTESUNATE/AMODIAQUINE AND COARTEM IN TREATMENT OF UNCOMPLICATED MALARIA IN PREGNANCY IN MULAGO HOSPITAL.
}

\author{
Kimera Lukanga Charles ${ }^{1 *}$ \\ ${ }^{* 1}$ Lecturer in department of Obstetrics and Gynaecology, School of Medicine, University of Namibia. Correspondent \\ email address: lkimera@unam.na
}

*Corresponding Author: -

Email address: lkimera@unam.na

\begin{abstract}
:
BACKGROUND: The therapeutic efficacy and safety of artesunate plus amodiaquine and artemether/lumefantrine were assessed in Mulago hospital, Kampala, Uganda, an area with high levels of Plasmodium falciparum resistance to chloroquine and sulphadoxinepyrimethamine.

MATERIALS AND METHODS: A total of 369 pregnant mothers beyond the first trimester (>12 weeks) and less than 37 weeks of gestation with signs and symptoms of malaria were screened for malaria parasites. Of these, 109 had uncomplicated malaria infection with parasite density 200 to 200,000 parasites/ $\mu L$ and were enrolled following informed consent. Eligible pregnant mothers were randomly assigned to receive either a 3-day course of

Coartem (Artemether 20mgs/ Lumefantrine 200mgs) 4 tablets twice a day 12 hourly on days 0, 1 and 2; or Artesunate/Amodiaquine on days 0, 1 and 2 (4tablets of Artesunate plus 4 tablets of Amodiaquine once a day). The Coartem arm had 55 participants while the Artesunat/Amodiaquine (DUACT) arm had 54 participants. Patients were followed up with clinical and laboratory assessments until day 14.

RESULTS: The clinical and parasitological response between Coarten (artemetherlumefantrine) and artesunate+amodiaquine (DUACT) in the treatment of uncomplicated malaria in pregnant mothers were statistically similar. The side effects for the two treatment arms were also statistically similar.

CONCLUSION: Artemether-lumenfantrine (coartem) and artesunate plus amodiaquine (DUACT) had high and similar cure rates and tolerability among pregnant mothers in Mulago Hospital, Uganda.
\end{abstract}

Key words: Malaria, Coartem, DUACT, Amodiaquine, pregnant women

Words Key: concepts of drugs emergent, new substances psychoactive, club drugs, synthesis drugs, intelligent drugs.

\section{(ㅇ) $(\$)$}




\section{INTRODUCTION:}

Malaria is an acute febrile illness characterized clinically by paroxysms of fever. These are the consequence of asexual reproduction by species of plasmodium falciparum within red blood cells. Malaria is transmitted by the bite of an infected female anopheles mosquito. It is always a serious disease and may be a deadly illness. According to the WHO world malaria report there were an estimated 219 million cases and 435000 related deaths in 2017 and most of these cases were in the WHO African Region (200 million or 92\%), followed by the WHO South-East Asia Region with 5\% of the cases and the WHO Eastern Mediterranean Region with $2 \%$ with Nigeria accounting for the highest percentage of cases $(25 \%)^{\mathrm{i}}$. Headache, anorexia, nausea, vomiting, body weakness and fever are prominent symptoms. Herpes simplex vesicles may appear on the lips during the illness.

Malaria is a preventable and treatable disease. Unfortunately the disease has continued to affect and kill people around the world with those in the tropics most affected.

The causative organism, the Plasmodium parasite, is transmitted by the blood sucking female anopheles mosquito which commonly feeds at night and in so doing transmits the organism from infected to uninfected individuals. Elimination of the disease therefore requires elimination of the transmission process and this requires prompt diagnosis and treated of infected individuals, preventing mosquitoes from reaching the infected and the uninfected individual, making mosquitoes incapable of harbouring the plasmodium or completely eliminating the mosquitoes by either removing the breeding sites, spraying with effective insecticides or use of biological methods.

Regarding prompt diagnosis and treated of infected individuals, malaria should always be considered among the differential diagnosis of a febrile illness in a patient who has traveled to an area where malaria is endemic. It should also be remembered that uncomplicated malaria can rapidly progress to severe forms of the disease especially in people with low or no immunity and severe falciparum malaria is almost fatal without treatment ${ }^{\mathrm{ii}}$.

Malaria infection during pregnancy is a major public health problem in tropical and subtropical regions throughout the world. In most endemic areas of Africa, pregnant women are the main adult risk group for malaria. The main burden of malaria infection during pregnancy results from infection with Plasmodium falciparum ${ }^{i i i}$.

Pregnant women, especially primigravid women, are up to 10 times more likely to contract malaria than non-gravid women. Gravid women who contract malaria also have a greater tendency to develop severe malaria. Unlike malarial infection in non-gravid individuals, pregnant women with $P$ vivax are at high risk for severe malaria, and those with $P$ falciparum have a greatly increased predisposition for severe malaria as well.

\section{Uncomplicated Malaria in Pregnancy}

In areas of stable malaria transmission, malaria illness is not significantly more severe during pregnancy. Symptoms include fever, headache, malaise, loss of appetite, general body weakness, cough, dizziness, nausea, vomiting, labour-like pains, joint pains, diarrhoea, muscle pains, backache, raised temperature, shivering, enlarged spleen, and a positive blood slide with parasite density of $200-200,000$ parasites/ $\square 1$

\section{Effects of Malaria on Pregnancy}

Studies have found that women have decreased resistance to malaria during pregnancy. That is why many pregnant women in Africa have malaria ${ }^{\text {iv }}$. Pregnant women are one of the vulnerable groups to malaria. Malaria in pregnancy carries significant morbidity and mortality for both the mother and the foetus ${ }^{v}$. For women in particular primigravidae and HIV positive women are at great risk for increased parasitemia and therefore anaemia, severe malaria and death. Malaria in pregnancy increases the chance of maternal anaemia, abortion, still births, prematurity, IUGR and LBW ${ }^{\mathrm{vi}}$. Severe anaemia increases risk for maternal mortality. Maternal anaemia is estimated to cause as many as 10,000 maternal deaths each year in Africa ${ }^{\text {vii }}$. If severe, anaemia puts women at risk of haemorrhage (PPH) and death. Maternal anaemia increases the risk of premature delivery and low birth weight babyviii. Most pregnant women with malaria do not have symptoms such that the mother may not even know that she is infected. However, even if an infected mother does not have fever, the baby is still at risk.

Overall, although the contribution of malaria in pregnancy to infant mortality may be modest, the wide geographic distribution of infection around the tropics and the high mortality rate in malaria-endemic settings leads to a substantial number of infant deaths linked to malaria in pregnancy.

According to WHO (1991), 60\% of primegravidae and 30\% of multigravidae in Africa are infected. During pregnancy, malaria episodes are more frequent and infections are more severe. Primegravidae have twice the rate of infection of multiparae perhaps because they lack the immunity that multiparae have developed in previous pregnancies ${ }^{\text {ix }}$.

\section{Effects of Pregnancy on Malaria}

Pregnant women attract more Anopheles gambiae mosquitoes, the major vector of malaria in Africa, than non-pregnant women under the same conditions yet pregnancy reduces a woman's immunity to malaria making her more susceptible to severe malaria than other adults ${ }^{\mathrm{x}}$.

Studies have shown the placenta to be an immunologically privileged site, which amplifies infection with malaria parasites. Placental malaria is recognized as a complication of malaria in pregnancy in areas of stable transmission and is particularly frequent and severe in prime gravidae (16\% to 60\%) whereas the prevalence is low in multigravidae (12 to $33 \%$ ). Placental malaria has been identified as a key factor for LBW. Plasmodium falciparum can adhere and massively sequester in the placenta and it has a tendency to multiply in the intervillous spaces. The mechanism for poor birth outcomes is due to placental parasite infection, which is associated with a local immune response including elevated proinflammatory cytokine levels and monocyte infiltration into the placental intervillous space. 


\section{Treatment of Uncomplicated Malaria}

Providing rapid diagnosis and treatment of malaria in pregnant women is an important component of effective control of malaria. There are a number of antimalarial drugs which are safe and effective for use during pregnancy.

WHO recommends a package of interventions for the prevention and control of malaria during pregnancy. This comprises of intermittent preventive treatment (IPT) to address the heavy burden of asymptomatic infections among pregnant women residing in areas of moderate to high transmission of plasmodium falciparum, use of insecticide treated net (ITN) and access to effective case management for malaria illness and anaemia. WHO recommends that at least two doses of SP are taken during regularly scheduled antenatal visits after the first trimester ${ }^{\mathrm{xi}}$.

Chloroquine was the antimalarial drug of choice for treatment of malaria for many years in most parts of the world. However Plasmodium falciparum became resistant to chloroquine first in Asia then Africa, making the drug ineffective against this dangerous Plasmodium strain in many affected regions of the world.

Combination based antimalarial formulations particularly Artemether/Lumefantrine are now being used for treatment of malaria in most parts of the world. In the United States, treatment options for uncomplicated chloroquine-resistant $P$ falciparum and $P$ vivax malaria in pregnant women are limited to mefloquine or quinine plus clindamycin. Although the limited availability of quinine and increasing resistance to mefloquine limit these options, strong evidence now demonstrates that artemether-lumefantrine (Coartem) is effective and safe in the treatment of malaria in pregnancy. These data are supported by the World Health Organization ${ }^{\mathrm{xii}}$.

Artemether-lumefantrine has also been recommended by $\mathrm{CDC}$ as an additional treatment option for uncomplicated malaria in pregnant women in the United States during the second and third trimester of pregnancy at the same doses recommended for non-pregnant women. During the first trimester of pregnancy, mefloquine or quinine plus clindamycin are used; however, when neither of these options is available, artemether-lumefantrine should be considered for treatment ${ }^{\mathrm{xii}}$

The main objective of this study was to compare the effectiveness and side effects of Artesunate/Amodiaquine with that of Coartem in the treatment of uncomplicated malaria in pregnancy in New Mulago Hospital, Uganda.

\section{MATERIALS AND METHOD:}

This work was carried out after getting the permission from institutional ethical committee i.e. the department of Obstetrics and Gynecology, Mulago hospital ethical review committee and the Uganda National Council of Science and Technology. Written informed consents were also obtained from participants. Participants were recruited on a daily basis till the required sample size was attained.

\section{Inclusion and exclusion criteria:}

Inclusion - pregnant women beyond gestation age 12 weeks presenting with axillary temperature $\square 37.5^{\circ} \mathrm{C}$ or a history of fever in the last 24 hours or at time of visit and having a positive blood film for malaria parasites $(200-2000000$ parasites/micro Liter).

Exclusion - women were ecluded from the study if they were known allergic to any of the study drugs e.g. Amodiaquine or if they had epetitive vomiting or if they had prior treatment with the study drugs or if their home was outside 10KM radius from hospital.

Mothers identified from their LNMP and clinical examination (Fundal Height) and counselled about participating in the study. Mothers were then screened for malaria parasites. Patients were then randomly allocated to either Coartem group or Artesunate/Amodiaquine group.

\section{Randomization procedure}

Participants were randomized by simple random sampling technique (drawing lots) in blocks of four (4) to receive either Coartem (artemether-lumefantrine) or artesunate+amodiaquine (DUACT). Allocation sequence was concealed in opaque, sealed envelopes which the participants had to draw from a box.

Blood smears to test for Malaria parasites were taken on the day of enrolment (Day 0). Thick blood smears were prepared and stained in $2 \%$ Giemsa solution for 30 minutes. The smears were read to 100 fields with quantification of $P$. falciparum asexual parasitaemia on the thick smear. Parasites were enumerated using thick film as described by Shute ${ }^{\mathrm{xiv}}$. The parasite density (per $\mu \mathrm{l}$ of blood) was calculated, assuming a normal leucocyte level of $8,000 / \mu l$. Patients with parasite count of $200-200,000$ parasites/ $\mu$ l were enrolled into the study. Study treatment was started on the day of randomization (day zero) and completed on day two, while follow-up continued for 14 days. Clinical examination findings were recorded on day zero. In one arm Coartem (Artemether $20 \mathrm{mgs} /$ Lumefantrine 200mgs) was given orally in a dose of 4 tablets twice a day 12 hourly on days 0,1 and 2 and in the arm Artesunate/Amodiaquine (DUACT) was given orally (4tablets of Artesunate plus 4 tablets of Amodiaquine) once daily on days 0,1 and 2. Treatment was started on the day of enrollment (Day 0). Treatment was directly supervised by research assistants and patients observed for 30 minutes. Drugs were readministered to those who vomited within this period. Tepid sponging, exposure and administration of paracetamol (1000mg 8hourly) were used to reduce high fever. Follow-up visits were scheduled on days 2, 7 and 14 . On each visit, clinical examination including axillary temperature and thick blood film specimen were obtained to screen for presence and density of malaria parasites. Patient addresses and telephone numbers were taken and participants that did not return on schedule for follow-up were communicated to on phone on the same day and provided information on their wellbeing. 
The following parameters were monitored:

1. Axillary temperature was measured on scheduled follow up days.

2. Parasite density- Blood slides for malaria parasites were taken and tested on days 0, 2,7 and 14.

3. Any symptoms assumed to be side effects of the treatments e.g. severe anaemia, itching, drowsiness were recorded on a separate form during the 14 days.

\section{Data Analysis}

Data generated were recorded in a log book, and individual patients case record files. Data were entered and analysed with EPI-Info version 6.4. Data were also exported to SPSS version 11.0 for further analysis. Differences between groups were assessed using chi-square

$\left(\mathrm{X}^{2}\right)$,'t' test for continuous normally distributed variables and nonparametric Kruskal-Wallis was used to compare continuous not normally distributed variables. The baseline characteristics in the two groups of Coartem and Artesunate/Amodiaquine were compared. Therapeutic efficacy was determined on day 14 or earlier by assessing both clinical and parasitological response based on the WHO guidelines for assessing therapeutic efficacy.

Analysis was by intention-to-treat principle.

\section{Results:}

A total of 369 mothers were screened and 109 eligible participants were enrolled. The coartem arm had 55 participants while the Artesunate/Amodiaquine (A/AD) arm had 54 participants (figure I \& II). Table I for baseline socio-demographic characteristics and Table II for obstetric characteristics \& prior treatment of the eligible participants show that the two groups (of coartem \& atesunate/amodiaquine) were comparable on baseline characteristics as there was no statistically significant difference in the characteristics of the two groups. Data on previous antimalarial drug-use pattern shows that $53(49.5 \%)$ of the enrolled participants had used antimalarial drugs in the past two weeks. The majority of these had used chloroquine and SP. Figure III shows the age distribution of the participants. Most of the participants (50.0\% of the Coartem group and $46.3 \%$ of the $\mathrm{A} / \mathrm{AD}$ group) were in the age range of $19-23$ years.

\section{Figure 1 Trial profile}

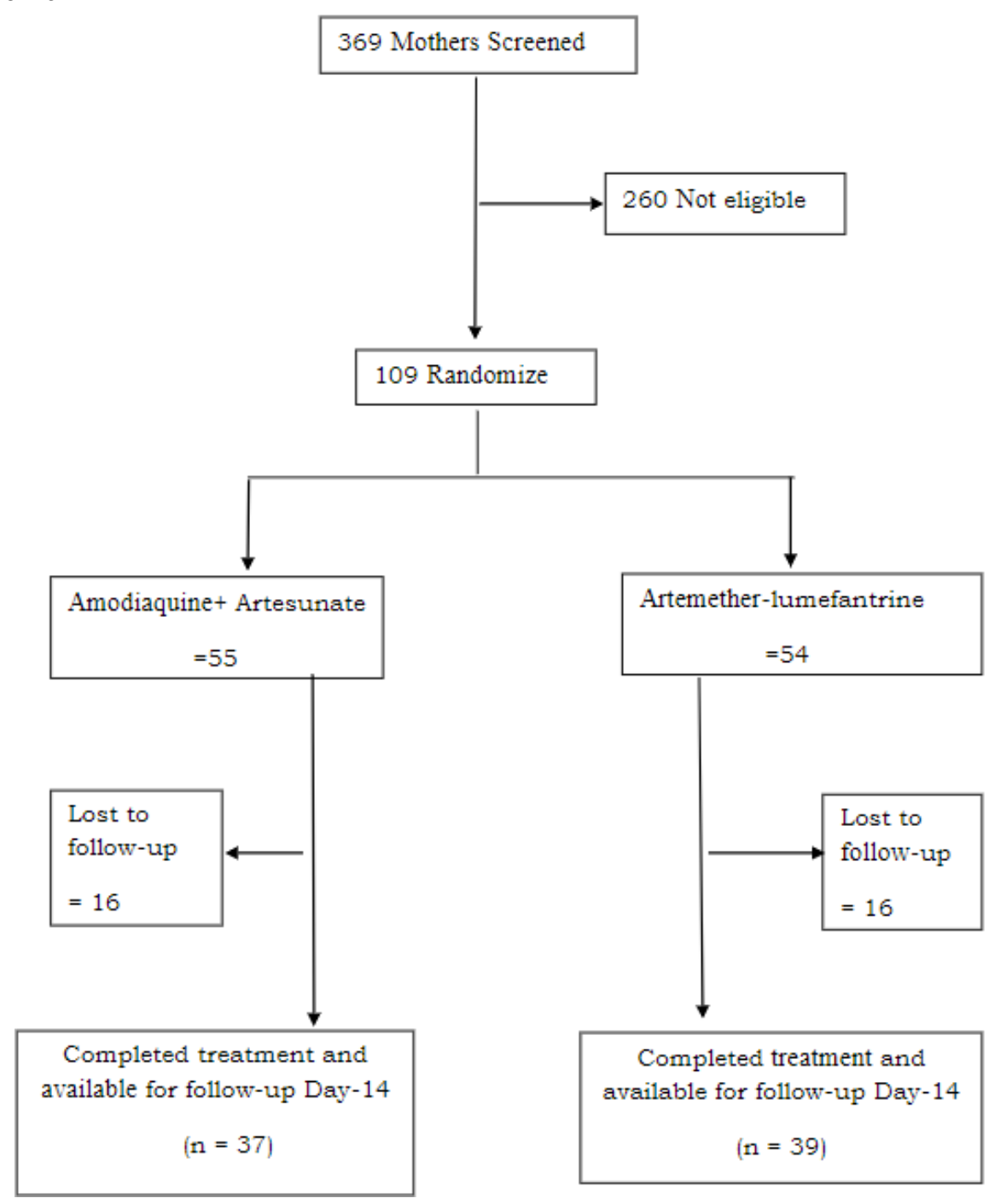


Figure II: RCT distribution of coartem vs artesunate/amoiaquine in treatment of uncomplicated malaria in pregnancy in Mulago Hospital
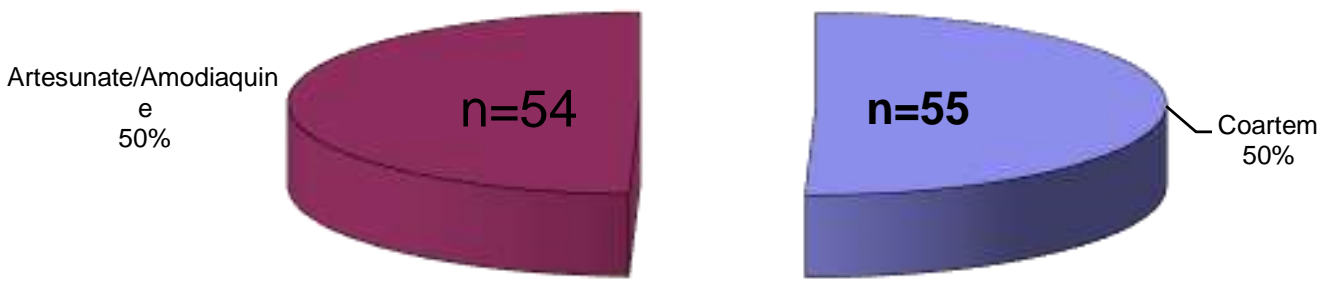

Figure III: Distribution of age of pregamnant women by treatment arm

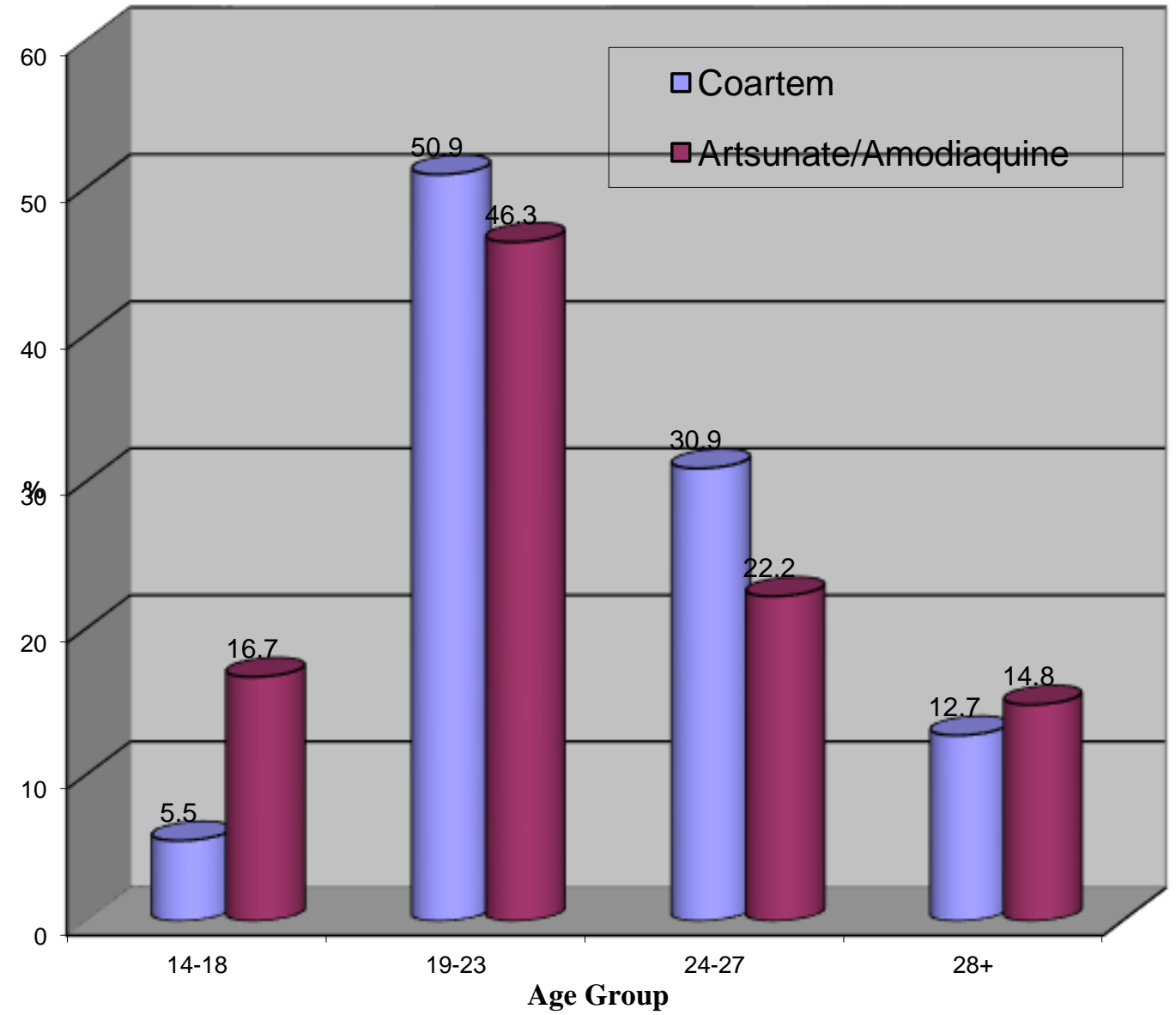


Table I: Obstetric Characteristics \& Prior Treatment of Pregnant Mothers by Treatment

\begin{tabular}{|c|c|c|c|c|c|}
\hline Characteristics & Coartem & $\begin{array}{l}\text { Artesunate/ } \\
\text { Amodiaquine }\end{array}$ & T-Test $\left(\chi^{2}\right)$ & $P$ value & OR \\
\hline \multicolumn{6}{|l|}{ LNMP/WOA } \\
\hline (Mean) & $28.6 \pm 5.4$ & $28.7 \pm 6.5$ & $t=-0.112$ & 0.911 & \\
\hline \multicolumn{3}{|l|}{ Gravidity } & $t=-0.289$ & & \\
\hline \multicolumn{6}{|c|}{ History of Abortion } \\
\hline Yes & $10(18.2)$ & $11(20.8)$ & \multirow[t]{2}{*}{$\chi^{2}=0.113$} & \multirow[t]{2}{*}{0.737} & 0.85 \\
\hline No & $45(81.8)$ & $42(79.2)$ & & & \\
\hline \multicolumn{6}{|c|}{ Numbers of Abortions } \\
\hline One & $7(87.5)$ & $9(75.0)$ & \multirow[t]{3}{*}{$\chi^{2}=0.688$} & \multirow{3}{*}{\multicolumn{2}{|c|}{0.407}} \\
\hline Two & $1(12.5)$ & $2(16.7)$ & & & \\
\hline Three & $0(0.0)$ & $1(8.3)$ & & & \\
\hline \multicolumn{6}{|c|}{ Number of children } \\
\hline One & $14(45.2)$ & $8(32.0)$ & \multirow{5}{*}{$\chi^{2}=0.462$} & \multirow{4}{*}{\multicolumn{2}{|c|}{0.496}} \\
\hline Two & $7(22.6)$ & $9(36.0)$ & & & \\
\hline Three & $7(22.6)$ & $4(16.0)$ & & & \\
\hline Four & $3(9.7)$ & $4(16.0)$ & & & \\
\hline \multicolumn{5}{|c|}{ Any Prior Treatment for the current illness } & \\
\hline Yes & $29(52.7)$ & $24(46.2)$ & \multirow[t]{3}{*}{$\chi 2=0.458$} & \multirow{3}{*}{\multicolumn{2}{|c|}{$\begin{array}{ll}0.499 & 1.30 \\
(\mathrm{CI} 0.60-2.78)\end{array}$}} \\
\hline No & $26(47.3)$ & $28(53.8)$ & & & \\
\hline \multicolumn{3}{|l|}{$\begin{array}{l}\text { No } \\
\text { Other drugs }\end{array}$} & & & \\
\hline Yes & $7(13.5)$ & $6(12.2)$ & \multirow[t]{2}{*}{$\chi 2=3.12$} & \multirow{2}{*}{\multicolumn{2}{|c|}{$\begin{array}{cc}0.539 & 1.15 \\
(\mathrm{CI} 0.35 & -3.58)\end{array}$}} \\
\hline No & $45(86.5)$ & $43(87.8)$ & & & \\
\hline
\end{tabular}

\section{** Fluctuating totals are due to missing Response}

There was no significant statistical difference in the weeks of amenorrhoea of the two study groups $(\mathrm{t}=-0.112$ $\mathrm{P}=0.911)$. Likewise, there was no statistical difference in the mean gravidity of the two study groups $(\mathrm{t}=-0.289$ $\mathrm{P}=0.773)$.

Considering the number of previous abortions, the participants had got, there was no significant statistical difference between the two groups ( $\square 2=0.688 \mathrm{P}=0.407$ ). Likewise, parity of the mothers was not statistically different between the two treatment groups ( $\square 2=0.462 \quad \mathrm{P}=0.496$ ).

There was no significant statistical difference between the two treatment arms as far as prior treatment for fever is concerned $\mathrm{p}=0.499 \mathrm{OR}=1.30(\mathrm{CI} 0.35-3.58)$.

Table II: Table for Screening Pregnant Mothers by Treatment

\begin{tabular}{|c|c|c|c|c|c|}
\hline & Crtoaem & $\begin{array}{l}\text { Artesunate/ } \\
\text { Amodiaquine }\end{array}$ & $\chi^{2}$ & $\mathrm{P}$ value & OR \\
\hline \multicolumn{3}{|c|}{ History of fever in the last 1 or 2 days } & \multirow[t]{3}{*}{0.00} & \multirow[t]{3}{*}{1.00} & 1.00 \\
\hline Yes & $53(98.1)$ & $53(98.1)$ & & & $(\mathrm{CI} 0.6-16.41)$ \\
\hline No & $1(1.9)$ & $1(1.9)$ & & & \\
\hline \multicolumn{6}{|c|}{ History of headache } \\
\hline Yes & $54(98.2)$ & $50(94.3)$ & \multirow[t]{2}{*}{1.107} & \multirow[t]{2}{*}{0.293} & 3.24 \\
\hline No & $1(1.8)$ & $3(5.7)$ & & & (CI $0.33-32.18)$ \\
\hline \multicolumn{6}{|c|}{ History of vomiting } \\
\hline Yes & $30(55.6)$ & $23(42.6)$ & \multirow[t]{2}{*}{1.799} & \multirow[t]{2}{*}{0.180} & 1.69 \\
\hline No & $24(44.4)$ & $31(57.4)$ & & & $(\mathrm{CI} 0.79-3.61)$ \\
\hline \multicolumn{6}{|c|}{ History of joint pain } \\
\hline Yes & $47(87.0)$ & $46(85.2)$ & \multirow[t]{2}{*}{0.077} & \multirow[t]{2}{*}{0.783} & 1.17 \\
\hline No & $7(13.0)$ & $8(14.8)$ & & & $(\mathrm{CI} 0.39-3.48)$ \\
\hline \multicolumn{6}{|c|}{ History of cough } \\
\hline Yes & $9(16.4)$ & $15(27.8)$ & \multirow[t]{2}{*}{2.049} & \multirow[t]{2}{*}{0.152} & 0.51 \\
\hline No & $46(83.6)$ & $39(72.2)$ & & & $(\mathrm{CI} 0.20-1.29)$ \\
\hline \multicolumn{6}{|c|}{ Severe pallor of mucous membranes } \\
\hline Yes & $2(3.6)$ & $5(9.3)$ & \multirow[t]{2}{*}{1.420} & \multirow[t]{2}{*}{0.233} & 0.37 \\
\hline No & $53(96.4)$ & $49(90.7)$ & & & $(\mathrm{CI} 0.07-2.00)$ \\
\hline \multicolumn{6}{|c|}{ Temperature on Day 0} \\
\hline$\geq 37.5^{\circ} \mathrm{C}$ & $44(83.0)$ & $37(72.5)$ & \multirow[t]{2}{*}{1.638} & \multirow[t]{2}{*}{0.201} & \\
\hline$<37.5^{\circ} \mathrm{C}$ & $9(17.0)$ & $14(27.5)$ & & & \\
\hline
\end{tabular}


Table II shows no significant statistical difference in the baseline screening characteristics of pregnant mothers between the two treatment arms.

Table IV: Mean parasite count \& side effects

\begin{tabular}{|c|c|c|c|}
\hline & Coartem & $\begin{array}{l}\text { Artesunate/ } \\
\text { Amodiaquine } \\
\end{array}$ & \\
\hline \multicolumn{4}{|c|}{ Mean Parasite counts at day 0} \\
\hline Side & $\begin{array}{l}27773.45 \pm 35621.03 \\
0 \text { to day } 14\end{array}$ & $33591.7 \pm 43766.35$ & $\begin{array}{l}t=-0.7590 \\
P=0.4495\end{array}$ \\
\hline Yes & $20(36.36)$ & $18(33.96)$ & $\chi 2=0.0682$ \\
\hline No & $35(63.64)$ & $35(66.04)$ & $P=0.794$ \\
\hline
\end{tabular}

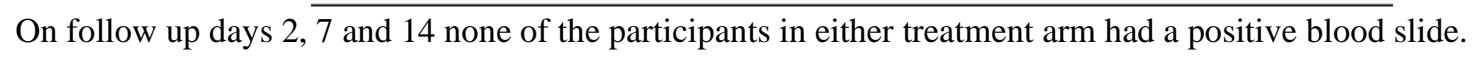

\section{Mean parasite count:}

There was no significant statistical difference in the mean parasite count on day 0 between the two treatment groups $(\mathrm{t}=$ $-0.7590 \mathrm{P}=0.4495)$. The Coartem group had a mean parasite count of $27773.45 \pm 35621.03$ while the A/AD group had a mean of $33591.7 \pm 43766.35$. From day 2 through day 14 of treatment all patients in both treatment arms had no parasite count (mean parasite count $=00000.00)$. So, in respect to parasite clearance, coartem and A/AD were equally efficacious.

\section{Side effects:}

The mothers who got side effects were equally distributed between the two treatment arms as shown in the table. There was no significant statistical difference in side effects of the two treatment arms $(\mathrm{P}=0.794)$. Throughout the course of treatment $20(36.36 \%)$ of the participants in the Coartem arm had reported some side effects compared to $18(33.96 \%)$ in the A/AD arm. The side effects reported were similar in the two treatment arms and included itching, dizziness, loss of appetite, nausea and body weakness.

Figure VI: Proportion of fever episodes against analysis time

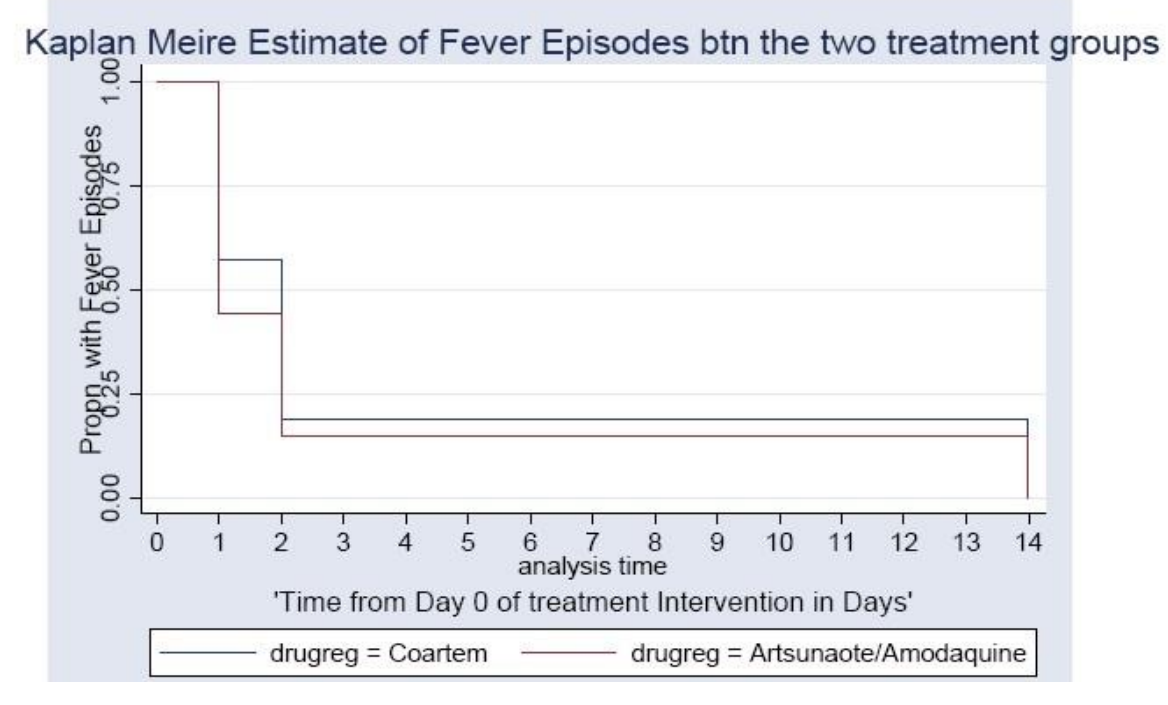

Log-rank test for equality of survivor functions chi2 $(1)=0.17 \mathrm{Pr}>\operatorname{chi} 2=0.6780$

On average Artesunate/Amodiaquine (A/AD) seemed to work much faster. By day one most of the fever had gone while the Coartem group took 2 days.

By Day one of treatment participants in A/AD treatment arm had less fever by self-reporting, whereas this happened on day 2 for the Coartem treatment arm. This is clinically significant. However for both treatment arms all participants had clinically less fever by day 2 of treatment indicating no significant statistical different between the two drug regimens ( $\mathrm{p}$ $=0.6780)$. 


\section{DISCUSSION}

Case management of malaria illness is an essential component of malaria prevention and control in all areas where pregnant women are at risk of malaria like in many countries in the tropics. Pregnant women with symptomatic malaria are at higher risk of foetal loss, premature delivery and death, and thus urgently need to be treated with an effective drug. Treatment of malaria in pregnancy aims at completely eliminating the infection because any level of parsitaemia may have adverse effects to the mother and the foetus. The effect of infection on the mother may range from negligible to severe, depending on the level of immunity to malaria infection that the mother has acquired prior to pregnancy and the efficacy of these immune responses during her pregnancy ${ }^{\mathrm{xv}}$.

Biomedically, today's malaria situation is precarious. The artemisinins are the only first-line antimalarial drugs appropriate for widespread use that still work against all chloroquineresistant malaria parasites. However artemisinins cost about 20 times more than chloroquine, and African countries and their citizens find it difficult to afford their higher price tag.

Artemisinin combination therapies (ACTs) were introduced to curb the increasing resistance of the malaria parasite to the existing first line and second line drugs (chloroquine and sulphadoxine-pyrimethamine). The key goal of ACTs is to enhance cure rates and delay development of parasite resistance to component drugs.

This study has shown rapid parasite and fever clearance in pregnant mothers at gestation age

12 - 37 weeks with 6-dose regimen of Coartem and 3-day course of Artesunate/Amodiaquine (DUACT). This is indicative that artesunate/amodiaquine is comparable to coartem in this aspect. This study also showed that these ACT regimens were similarly tolerable among pregnant mothers in Mulago national referral hospital in Kampala, Uganda $(\mathrm{p}=0.794)$. During the period of treatment none of the mothers complained of unique side effects that could mean severe adverse effects; there were no abortions/miscarriage and there were no reported intrauterine foetal deaths following commencement of treatment. This suggests that the ACTs in the study were well tolerated among pregnant mothers in addition to being effective.

It is, however, important to note that DUACT had faster fever clearance which is of much clinical benefit among pregnant mothers since high temperatures are the main contributor to both intrauterine foetal death (IUFD) and abortions. By day 1 of treatment there was $50 \%$ reduction in fever with DUACT. However there was no statistical difference in respect to fever between the two treatment arms by day $2(\mathrm{p}=0.678)$.

These results mean that:

- The two drug regimens (coartem and DUACT) are equally effective in respect to parasite clearance, symptom clearance and tolerability.

- The choice of which drug regimen to use in pregnant women should depend on the cost and other features rather than efficacy.

In support to safety McGreagy et $\mathrm{al}^{\mathrm{xvi}}$ in a detailed follow up on efficacy and safety (including follow up of infants until 1 year of life) in Asia and Gambia found that a single dose of the combination of artesunate and sulfadoxinpyrymethamine (SP) during pregnancy was safe. They also concluded from the available evidence that artemisinins are at least as safe as Quinine, Chloroquine and SP for treatment of falciparum malaria in pregnant women in the $2^{\text {nd }}$ and $3^{\text {rd }}$ trimester of pregnancy.

\section{Study Limitations}

1. Mothers were followed up for 14 days rather than 28 days, the period for which a patient can be declared to be malaria free.

2. It was not possible to examine placentas of mothers in the study for parasite clearance to determine long-term protection from malaria.

3. PCR was not done to specifically determine the plasmodium parasites.

\section{Applicability of the results}

The results of this study are particularly important to policy makers. Now that it has been shown that the two drug regimens are comparable in efficacy, the onus remains to the policy makers to try and find out which regimen is affordable either by the concerned government or the local population.

\section{Conclusion}

It is important that early and correct treatment of malaria in pregnancy is observed among our local population. This combines early diagnosis from symptoms \& signs and laboratory investigations and prescribing the correct dosage of an effective drug. Coartem and DUACT have been shown by this study to be equally effective in parasite clearance, both have fast symptom clearance and tolerable in the treatment of malaria among pregnant mothers.

[1].WHO world malaria report 2018

[2].WHO malaria treatment guidelines 2015

[3].Africa Malaria Report, 2003 - The World Health Organisation

[4].Steketee RW, Nahlen BL, Parise ME, Menedez C. The burden of malaria in pregnancy in malaria-endemic areas. American journal of tropical medicine and hygiene 2001 ; 64(1, 2) S: 28-35.

[5].Centre for Disease Control and Prevention (CDC) 
[6]. Menendez C ${ }^{1}$, Ordi J, Ismail MR, Ventura PJ, Aponte JJ, Kahigwa E, Font F, Alonso PL: The impact of placental malaria on gestational age and birth weight.

[7].Malaria Prev. new - Regional Office for Africa - World Health https://www.afro.who.int/sites/default/files/2017.../malaria_in_pregnancy_092004

[8].Richard W. Steketee, Bernard L. Nahlen, Monica E. Parise, and Clara Menendez: The Burden of Malaria in Pregnancy in Malaria-Endemic Areas

[9].Tanzania Health Research Bulletin (2007), Vol. 9, No. 3: Placental malaria in Owerri, Imo State, south-eastern Nigeria

[10]. Effect of pregnancy on exposure to malaria mosquitoes. - NCBI - S Lindsay - $2000{ }^{\text {xi }}$ WHO | Intermittent preventive treatment in pregnancy (IPTp) https://www.who.int/malaria/areas/preventive_therapies/pregnancy/en/

[11]. Malaria Treatment and Management: https: emedicine.mediscape.com

[12]. Centres for Disease Control and Prevention. Updated CDC Recommendations for Using ArtemetherLumefantrine for the Treatment of Uncomplicated Malaria in Pregnant Women in the United States; https://www.cdc.gov/mmwr/volumes/67/wr/mm6714a4.htm?s_cid=mm6714a4_e\#contribAff. April 2018; Accessed: April 13, 2018.)

[13]. Wernsdorfer, McGregor L. Edinburgh: Churchhill Livingstone; 1988:781- 814. Shute GT: The microscopic diagnosis of malaria. In Principle and practice of malariology

[14]. Steketee RW et al. the problem of malaria and malaria control in pregnancy in sub-Saharan Africa. American journal of tropical medicine and hygien, 1996, 55 (1 suppl), 2-7.

[15]. McGready R, Cho, T, Keo, NK, et al. Artemisinin antimalarials in pregnancy: a prospective treatment study of 539 episodes of Multidrug-resistant Plasmodium falciparum. Clin Infect Dis 2001;33:2009. 